\title{
Especificaciones del servicio de medición y control de la presión arterial mediante MAFC, AMPA y MAPA
}

\author{
Elaborado por \\ Comisión Servicios Profesionales Farmacéuticos SEFAC \\ Revisado por \\ Oscar Penín, Eduardo Satué, Salvador Tous, Nina Villasuso, Vicente J. Baixauli, Ana Molinero \\ Grupo de trabajo de Hipertensión arterial y riesgo vascular de SEFAC
}

\section{PALABRAS CLAVE}

Hipertensión arterial, riesgo cardiovascular, medida de la presión arterial, farmacéutico comunitario, farmacia comunitaria, servicios profesionales farmacéuticos, retribución

\section{ABREVIATURAS}

AMPA: automedida de la presión arterial AVAC: años de vida ajustados a calidad GIAF-UGR: Grupo de Investigación en Atención Farmacéutica de la Universidad de Granada

HTA: hipertensión arterial

IRC: insuficiencia renal crónica

LOPD: Ley Orgánica de Protección de Datos

MAFC: medida aislada en farmacia comunitaria

MAPA: medida ambulatoria de la presión arterial

NICE: The National Institute for Health and Care Excellence

PA: presión arterial

PNT: procedimiento normalizado de trabajo

RCV: riesgo cardiovascular

RUM: revisión de uso de los

medicamentos

SEFAC: Sociedad Española de Farmacia

Familiar y Comunitaria

SEH-LELHA: Sociedad Española de

Hipertensión-Liga Española para la Lucha contra la Hipertensión Arterial

SPD: sistema personalizado de dosificación

SPF: servicios profesionales

farmacéuticos

ZAP: zona de atención personalizada

\section{KEYWORDS}

High blood pressure, cardiovascular risk, measurement of blood pressure, community pharmacist, community pharmacy, professional pharmaceutical services, remuneration

Recibido: $10 / 3 / 2016$

Aceptado: 24/5/2016

Disponible online: 1-6-2016

\section{RESUMEN}

En abril de 2013, la Sociedad Española de Farmacia Familiar y Comunitaria (SEFAC) presentó su propuesta sobre servicios profesionales farmacéuticos (SPF) cuyo fin es cubrir las necesidades relacionadas tanto con la atención de los pacientes que utilizan medicamentos, como con la salud pública. Esta propuesta ofrece un planteamiento sobre la implantación y desarrollo de los SPF con el objetivo de impulsar su prestación por las farmacias comunitarias en los próximos años. De acuerdo con dicha propuesta todos los SPF que constituyen el catálogo de servicios contarán con un documento de especificaciones.

El objeto de este documento de especificaciones es definir y caracterizar el servicio de medición y control de la presión arterial con un doble objeto:

- Ayudar al farmacéutico comunitario y a sus representantes en el ofrecimiento, prestación, difusión, financiación y concertación de este servicio.

- Servir de guía a los farmacéuticos comunitarios que desean implantar este servicio en la farmacia o elaborar un procedimiento normalizado de trabajo para su realización.

Este documento se complementa con la Guia de actuación para el farmacéutico comunitario en pacientes con HTA y riesgo cardiovascular (RCV). Documento de consenso GIAF-UGR, SEFAC y SEH-LELHA y con el programa impacHta: formación de SEFAC y SEH-LELHA en hipertensión y riesgo vascular.

Specifications of the arterial pressure measurement and testing service using one-off measurement in community pharmacies, arterial pressure self-measurement and outpatient arterial pressure monitoring

\section{ABSTRACT}

In April 2013, the Spanish Family and Community Pharmacy Society (Sociedad Española de Farmacia Familiar y Comunitaria - SEFAC) issued a proposal for professional pharmacy services, with an aim to cover both the needs relating to care for patients on medication and to public health. This proposal put forward an approach for the implementation and development of professional pharmacy services with an aim to roll out this service in community pharmacies in the next few years. In accordance with this proposal, all professional pharmacy services that offer these services will be supported by a specifications document.

The purpose of this specifications document is to define and characterize the arterial pressure measurement and testing service with two aims:

- To help community pharmacists and their representatives in the offer, provision, promotion, financing and arrangement of this service.

- To serve as a guide for community pharmacists who want to implement this service in the pharmacy or to provide a standardized working procedure to do so.

This document is accompanied by the procedural guide for community pharmacists for patients with arterial hypertension and cardiovascular risk (Guia de actuación para el farmacéutico comunitario en pacientes con HTA y riesgo cardiovascular (RCV)). Consensus document of GIAF-UGR, SEFAC and SEH-LELHA and the impacHta program: SEFAC and SEH-LELHA training in hypertension and vascular risk.

Financiación: Ninguna ajena.

Conflicto de intereses: Los autores declaran no existir conflicto de intereses en relación con el contenido del presente artículo.

Cite este artículo como: Comisión Servicios Profesionales Farmacéuticos SEFAC. Especificaciones del servicio de medición y control de la presión arterial mediante MAFC, AMPA y MAPA. Farmacéuticos Comunitarios. 2016 Jun 30; 8(2):16-23. doi:10.5672/FC.2173-9218.(2016/Nol8).002.03

Correspondencia: sefac@sefac.org

ISSN 1885-8619 @ SEFAC (Sociedad Española de Farmacia Familiar y Comunitaria). Todos los derechos reservados. 


\section{Introducción y justificación}

La Sociedad Española de Farmacia Familiar y Comunitaria (SEFAC) en su propuesta sobre servicios profesionales farmacéuticos (SPF) [1] se compromete a impulsar la implantación y desarrollo de los SPF con el objetivo de facilitar su implantación por las farmacias comunitarias. La hipertensión arterial (HTA) es uno de los factores de riesgo vascular más importantes y se relaciona con un elevado porcentaje de muertes por enfermedades coronarias, cerebrovasculares [2,3], etc.

En la actualidad, el control y seguimiento del paciente hipertenso, si bien ha mejorado algo en los últimos años, sigue siendo insuficiente y se hace necesario mejorar la detección precoz del problema y en su caso la seguridad y efectividad de los tratamientos, sobre todo en pacientes con multimorbilidad [4] como los que proponen las guías clínicas de HTA más relevantes a escala internacional (NICE 2011) [5], Guía europea para el manejo de la hipertensión arterial 2013) [6], para el diagnóstico, control y seguimiento. A su vez, cobra una importancia especial la irrupción de la receta electrónica en prácticamente todo el sistema sanitario español, que permite que el médico pueda cargar la medicación hasta un año. Esta situación provoca que el paciente ya no tenga que acudir al centro de salud con tanta frecuencia, con el riesgo que esto conlleva puesto que puede disminuir el control de valores tensionales.

En este marco, el farmacéutico puede mostrarse como una pieza muy accesible al paciente con el fin de realizar un control y seguimiento de este factor de riesgo [7]. Sin embargo, cualquier control debe estar basado en protocolos consensuados por la comunidad científica y preferiblemente certificados por entidades acreditadoras válidas.

La hipertensión se conoce como la enfermedad de los tercios ya que:

- 1 de cada 3 pacientes sufre hipertensión.

- 1 de cada 3 pacientes con hipertensión desconoce que tiene la enfermedad.

- 1 de cada 3 pacientes que conoce su hipertensión no la tiene controlada.

La epidemiología de HTA en España [8] se refleja en la tabla 1.
Este elevado porcentaje, sobre todo en pacientes mayores de 60 años, muestra que existe una necesidad de trabajo sanitario a todos los niveles: detección, educación, control y seguimiento en el que el farmacéutico puede realizar un papel complementario con otras profesiones sanitarias.

Las recomendaciones establecidas en el documento de consenso español para la evaluación y tratamiento de HTA [9], así como las guías nacionales [2] e internacionales [6] sugieren no basar la detección de HTA, así como su control y seguimiento, únicamente en la medición de la presión arterial (PA) aislada en consulta o medición aislada en farmacia comunitaria (MAFC), sino en el empleo de un método complementario, Auto Medida de la Presión Arterial (AMPA) o Medida Ambulatoria de la Presión Arterial (MAPA), que favorezca la precisión del diagnóstico de HTA, así como su control y seguimiento. Por otra parte, existe una prevalencia variable de hipertensión de bata blanca o hipertensión aislada en la consulta (elevación de las cifras de PA en consulta frente a valores normales fuera de la misma obtenidos con AMPA o MAPA) alrededor de un 13\% y de hipertensión enmascarada (hipertensión con valores normales en consulta pero elevados fuera de ella) también alrededor de un 13\%, que refuerzan la necesidad del uso de estas técnicas complementarias para mejorar el conocimiento y en lo posible el grado de control de la hipertensión [6].

El presente documento, complementa a la Guía de actuación para el farmacéutico comunitario en pacientes con HTA y riesgo cardiovascular (RCV). Documento de consenso GIAF-UGR, SEFAC y SEH-LELHA y al programa impacHta: formación SEFAC y SEH-LELHA en hipertensión y riesgo vascular [10] y pretende así favorecer la implantación del servicio de control de la presión arterial en sus diferentes modalidades en la farmacia comunitaria.

Tabla 1 Prevalencia de la HTA en España

\begin{tabular}{|c|c|}
\hline Prevalencia & Intervalo etario \\
\hline $10 \%$ & $20-40$ años \\
\hline $50 \%$ & $50-60$ años \\
\hline $68 \%$ & $>60$ años \\
\hline
\end{tabular}

\section{Definición y descripción breve}

Este servicio consiste en la medida y el control de la PA del paciente, ya sea en la farmacia comunitaria o en el domicilio del paciente. La medida de la PA puede realizarse desde la farmacia comunitaria, a través de distintos métodos de medición validados en función de las características y necesidades del paciente, mientras que el control se realiza a través del seguimiento de los valores de PA obtenidos respecto de los objetivos de control de ese paciente, el asesoramiento farmacéutico al paciente y la colaboración con el resto de profesionales sanitarios.

Los métodos de medida consisten en:

Medida aislada de PA (MAFC): medida realizada en la farmacia comunitaria con arreglo a las guías internacionales mediante un tensiómetro oscilométrico clínicamente validado y calibrado con hasta tres tomas en caso necesario separadas por dos minutos.

Automedida de la PA (AMPA): medidas triples de la PA mediante tensiómetro oscilométrico clínicamente validado y calibrado, por la mañana y por la tarde, durante varios días seguidos, generalmente en el domicilio o ambiente cotidiano del paciente y ejecutado por él mismo (o por un cuidador entrenado) previa instrucción de manejo en la farmacia.

Medida ambulatoria de la PA (MAPA): medidas continuadas de la PA mediante tensiómetro automatizado clínicamente validado y calibrado, cada 15-30 minutos, durante 29/48 horas seguidas (incluida la noche) generalmente en el domicilio del paciente sin que sea necesaria la intervención del mismo.

El farmacéutico comunitario ha de establecer protocolos para cada uno de estos métodos que aseguren la correcta realización de los mismos, así como una intervención de seguimiento y control de la PA sobre el paciente que pueden derivar en informes a otros profesionales sanitarios como, por ejemplo, el médico de AP, el cardiólogo, nefrólogo, etc.

\section{Objetivos}

Objetivos generales

- Determinar la PA de los pacientes y usuarios. 
- Mejorar el grado de control de la PA de los pacientes atendidos.

- Asesorar e implicar a los pacientes en el control de su PA.

\section{Objetivos específicos}

- Mejorar el conocimiento del paciente sobre los estilos de vida saludables, medidas higiénico-dietéticas y farmacoterapéuticas relacionadas con la hipertensión.

- Realizar informes estandarizados con los resultados obtenidos mediante MAFC, AMPA y MAPA que podrán ser utilizados para su posterior valoración por parte del médico u otros profesionales sanitarios.

- Colaborar con el médico u otros profesionales de la salud que atiendan al paciente en el control de la farmacoterapia antihipertensiva.

Como objetivos específicos concretos de cada método de medida encontramos:

MAFC: determinar de forma aislada la PA en farmacia comunitaria que puede servir para detectar una posible situación de hipertensión o para derivar al médico según la urgencia. En definitiva permite obtener una primera aproximación al grado de control de PA del paciente.

Comunes para AMPA y MAPA: conocer la PA del paciente en un contexto más próximo a las condiciones de vida cotidiana y en particular en:

AMPA: determinar el estado de PA diurna media del paciente.

MAPA: determinación exhaustiva de los valores de PA tanto diurnos como nocturnos, la carga global de presión arterial, la cantidad de descenso durante el sueño, el incremento matutino de la PA, y la variabilidad de la PA, que pueden influir en el perfil de riesgo del paciente.

\section{Población/ pacientes diana}

La población diana como tal podría ser cualquier paciente o usuario que acude a la farmacia, sea o no hipertenso, ya que un buen control de las cifras tensionales disminuye la gravedad de otras patologías asociadas a la hipertensión. Al mismo tiempo, ciertas patologías que puedan estar presentes (hipercolesterolemia, hiperuricemia, diabetes, etc.), servirian de indicadores de qué pacientes necesitarian un mayor control de los valores de PA. Por lo tanto, los candidatos a incluir en un servicio de control de la PA serán aquellos que:

- Ya están diagnosticados de hipertensión y en tratamiento y pueden beneficiarse de un control y seguimiento en la farmacia a fin de determinar la efectividad de su tratamiento.

- No padecen hipertensión pero sufren otras patologías que pueden agravarse con ella, como, por ejemplo, la insuficiencia renal, hiperuricemia, apnea, disfunción eréctil, enfermedad coronaria, insuficiencia cardiaca, etc., por lo que un seguimiento preventivo ayudaría al control de su enfermedad primaria.

- Pacientes varones mayores de 55 años y mujeres mayores de 65 años que no tengan constancia de haberse controlado la PA en el último año.

- Cualquier paciente que sin estar en los dos grupos anteriores presente indicios de que pueda padecer hipertensión: mareos, dolor de cabeza... o estar en grupos habitualmente asociados a hipertensión: antecedentes familiares, diabéticos, dislipémicos, obesos...

- Pacientes que presenten gran variación en las medidas de tensión arterial o que manifiesten padecer hipertensión de bata blanca.

En función de la situación y de las necesidades de cada paciente o usuario se utilizará preferiblemente uno $u$ otro método de medida o modalidad de servicio de control de la PA:

\section{MAFC: toda la población y}

especialmente:

- Hipertensos diagnosticados y tratados.

- Normotensos con patologías que incrementan riesgo vascular: diabetes, insuficiencia renal crónica (IRC), etc.

- Pacientes varones mayores de 55 años y mujeres mayores de 65 años, al menos una vez al año.

- Usuarios con sintomatología que pueda relacionarse con HTA (mareos, cefalea...).

\section{AMPA:}

- Sospecha de hipertensión de bata blanca.
- Sospecha de hipertensión enmascarada.

- Alta variabilidad de PA en MAFC.

- Hipotensión arterial.

- Embarazada con PA>140/90.

- Sospecha de HTA resistente a fármacos.

MAPA: igual que en AMPA y además:

- Alta discordancia entre MAFC y AMPA.

- Identificación de patrón nocturno de PA:

- Descenso normal (dipper). 10$20 \%$ de diferencia porcentual entre PA en el sueño y PA en vigilia.

- Descenso insuficiente (non-dipper). 0-10\% de diferencia.

- Elevación (riser) $\quad<0 \%$ de diferencia.

- Descenso extremo (dipper extremo). > 20\% de diferencia.

- Sospecha de HTA nocturna (en apnea del sueño, IRC o diabetes)

- Evaluar variabilidad de PA a lo largo del día.

\section{Recursos necesarios}

Recursos materiales

1. Zona de Atención Personalizada (ZAP).

En el servicio de AMPA/MAPA se requiere una interrelación entre el farmacéutico y el paciente. La confianza y la confidencialidad son pilares básicos, por lo que es imprescindible disponer de una zona diferenciada y distinta de la zona de dispensación, pero que sea visible desde ésta y fácilmente accesible, que garantice la intimidad (el paciente y el farmacéutico puedan hablar sentados sin ser escuchados por cualquier otra persona, incluyendo el personal que trabaja en la farmacia), la relajación del paciente y que cumpla con las condiciones que debe reunir éste para obtener la medida basal de PA [2]. La ZAP facilita que el paciente se relaje, lo que indica su utilidad en todas las modalidades del servicio de medición y control de la PA. Debe estar provista de una mesa amplia, sillas y el resto de recursos materiales necesarios para prestar el servicio. Además hay que tener en cuenta que en el caso de la MAPA, la colocación del tensiómetro habitualmente precisa que el paciente se quede en ropa interior, por lo que habrá que garantizar su intimidad. 
Dependiendo de la carga asistencial puede también ser conveniente la habilitación de una zona de espera para los pacientes de la ZAP.

\section{Materiales impresos:}

a) Cartelería (opcional).

b) Folletos educativos para los pacientes.

c) Hojas de registro, (medidas de PA, test para valorar cumplimiento...).

\section{Documentación y registros:}

a) Ficha de cada paciente (consentimiento informado, incluirá registros de PA, datos de medicación antihipertensiva...).

b) Informes dirigidos al médico.

Se procederá a registrar los datos relativos a las enfermedades de riesgo vascular o relacionados con la hipertensión, como diagnóstico que se le ha efectuado, datos de control de PA anteriores, tratamiento antihipertensivo... por lo que es necesario disponer de un sistema de registro seguro de toda la información y documentación utilizada. Se recomienda disponer de ellos en formato digital para su mejor cumplimentación, archivo y recuperación.

Hay que distinguir entre la información procedente de los registros de dispensación u otros servicios de la propia farmacia de aquella procedente de otros profesionales sanitarios (informes médicos y pruebas analíticas que aporten los pacientes para complementar la historia farmacoterapéutica, informes de alta hospitalaria de los pacientes, etc.).

4. Fuentes de información sobre gestión de riesgo vascular y tratamientos.

Es preciso acceder a las guías clínicas de manejo de la HTA [6] o de riesgo vascular [11], con el fin de actualizar datos, protocolos, etc. como la Guía de actuación para el farmacéutico comunitario en pacientes con HTA y riesgo cardiovascular [2], que reúne la mayor parte de la información necesaria para realizar un correcto control de la PA del paciente.

Asimismo, es conveniente disponer de acceso a fuentes de información sobre medicamentos como la base de datos del Consejo General de Colegios Oficiales de Farmacéuticos (BOTPlus ${ }^{\circledR}$ ) [12].

\section{Utillaje}

- Ordenador con acceso a Internet e impresora.
- En función de la modalidad del servicio:

- MAFC: tensiómetro clínicamente validado.

- AMPA: tensiómetro clínicamente validado, preferentemente capaz de realizar 2 o 3 medidas seguidas. Puede utilizarse para realizar MAFC.

- MAPA: tensiómetro clínicamente validado automatizado y programable.

- Manguitos de diferentes medidas.

- Pilas.

\section{Recursos humanos}

Los recursos humanos son el factor determinante a la hora de desarrollar la AMPA/MAPA, dado que se trata de un servicio profesional donde la preparación y el tiempo de los profesionales resultan imprescindibles. Por ello todos los farmacéuticos que presten este servicio deberán estar capacitados específicamente para ello.

Responsable del servicio: con el fin de organizar mejor este servicio es conveniente que exista un farmacéutico responsable del servicio. Cuando en la farmacia sólo trabaja un farmacéutico éste será el responsable del servicio. Si trabaja más de un farmacéutico se debe nombrar un responsable entre todos los farmacéuticos del equipo. La elección del farmacéutico responsable del servicio se debería tomar en función de las habilidades de comunicación, la actitud frente al servicio y la experiencia asistencial. El responsable del servicio, junto con el farmacéutico titular, debería marcar los objetivos e indicadores del servicio, con el fin de evaluarlos cada año junto a la satisfacción de los pacientes.

Continuidad del servicio: para facilitar la continuidad del servicio a lo largo del tiempo es aconsejable que todos los farmacéuticos de la farmacia estén capacitados para poder prestarlo, así se evita su paralización ante periodos de vacaciones, bajas por enfermedad o ausencia del responsable de la farmacia.

Obligaciones del farmacéutico responsable del servicio: el farmacéutico responsable debe asumir ciertas tareas específicas relacionadas con la provisión del servicio.

Personal técnico: el personal técnico debidamente adiestrado por el farmacéutico podrá auxiliar al farmacéutico responsable en diversas tareas adecuadas a su categoría como por ejemplo anotar el dato de la tensión, enseñar a colocar el manguito, descargar datos en el ordenador, etc.

\section{Recursos temporales}

AMPA y MAPA son actividades clínicas que obligan a poner y retirar el aparataje pasadas 29/48 horas en el caso de MAPA o enseñar a manejar el tensiómetro en caso de AMPA, así como tomar decisiones respecto al paciente que utiliza medicamentos, lo que implica estudiar el caso, preparar las entrevistas y las intervenciones y realizar los registros correspondientes de la actividad, lo que supone un consumo de tiempo importante. A modo de ejemplo, el estudio del caso puede conllevar sugerencias al médico para cambiar pauta de administración, posología, dosificación, determinación de factores de riesgo como ser non-dipper, riser...

\section{Derivación}

En el transcurso del servicio, el farmacéutico puede detectar situaciones que excedan los objetivos del mismo y precisen de una intervención profesional adicional. En estos casos, el farmacéutico indicará al paciente la conveniencia de derivarle a otro servicio, en el que se aborde la situación detectada.

La derivación quedará reflejada en el informe del servicio, así como en el procedimiento normalizado de trabajo (PNT) correspondiente, donde se especificarán las situaciones y circunstancias que la determinen. La derivación puede ser de dos tipos: interna y externa.

\section{Derivación interna}

La situación detectada puede tratarse mediante otro servicio disponible en la misma farmacia comunitaria, sea por el mismo profesional $u$ otro del establecimiento. Se registrará el problema, el servicio interno al que se deriva y la justificación.

Los casos más habituales de derivación interna en este servicio pueden ser:

- Malos hábitos higiénico-dietéticos: puede necesitarse un servicio de educación para la salud, de dietética, de cesación tabáquica... 
- Problemas en la farmacoterapia: requerirá la puesta en marcha de un servicio de revisión farmacoterapéutica e incluso de seguimiento farmacoterapéutico.

- Problemas en la adherencia al tratamiento o dificultad en la utilización de los medicamentos: precisará de un servicio de revisión de uso de los medicamentos (RUM), sistemas personalizados de dosificación (SPD), formulación magistral...

\section{Derivación externa}

La situación detectada no puede tratarse en la propia farmacia, por no prestar dicho servicio, exceder las competencias del personal farmacéutico de dicha farmacia o requerir de otro nivel asistencial, por lo que es preciso derivar a otro profesional sanitario. Igualmente se realizará un parte de derivación, previo consentimiento del paciente, adjuntando toda la información necesaria para el otro profesional.

El caso más habitual de derivación externa en este servicio es:

- Problemas farmacoterapéuticos, que afecten directamente al tratamiento y precisen ser modificados por el médico responsable del paciente.

\section{Protocolización}

El servicio de control de PA deberá estar protocolizado y el procedimiento normalizado de trabajo deberá ser seguido y conocido por todo el equipo de la farmacia.

El PNT es una herramienta para el profesional, por lo que debe adaptarse a la situación concreta y específica de los profesionales que ejercen en dicha farmacia. Por tanto, sería conveniente que los farmacéuticos de cada farmacia desarrollasen su propio PNT, como por ejemplo partiendo de PNT estándares [9] y amoldándolos a la situación particular.

En los casos de AMPA y MAPA cabe destacar que tendremos dos protocolos para cada uno. Uno para los farmacéuticos y otro que han de cumplir los pacientes para que las tomas sean válidas y reflejen lo más fielmente posible la situación del paciente. Asimismo, los informes que se generen deberán seguir unas pautas de rigor y claridad a fin de ofrecer la máxima información posible.

\section{Documentación y registros}

La documentación del servicio AMPA y MAPA está constituida principalmente por:

- Consentimiento informado para la prestación del servicio que incluirá el consentimiento para el tratamiento de datos personales de acuerdo con la Ley de Protección de Datos (LOPD).

Es necesario pedir autorización firmada al paciente. Una vez firmada la autorización por el farmacéutico y por el paciente se le entregará una copia de este documento. Si un paciente se niega a dar su consentimiento, el farmacéutico no puede prestarle el servicio AMPA o MAPA.

Normalmente en el mismo consentimiento se incluye la posibilidad de proporcionar la autorización del paciente para que el farmacéutico comparta información sobre la intervención con médicos $\mathrm{u}$ otros proveedores de atención médica, cuando ésta sea necesaria.

En el caso de MAFC, si no se guardan datos personales, bastará con un consentimiento verbal. En situaciones excepcionales el consentimiento verbal podrá servir también para realizar AMPA o MAPA pero no es lo recomendable, sobre todo si hay conservación de datos personales.

\section{- Registros:}

El registro de los datos obtenidos permite realizar un informe que será evaluado por el médico que tomará las acciones oportunas. En función de ello y realizando un nuevo control posteriormente, siempre que hubiese cambios en la medicación: tipo de medicamento, dosis o pauta, obtendremos unos resultados que permiten demostrar el impacto de la actuación farmacéutica en el paciente. El registro podrá realizarse en soporte papel o preferiblemente en soporte informático garantizando la confidencialidad y seguridad de los datos, adaptándose a la LOPD.

Los registros necesarios para el servicio AMPA/MAPA deben contener fundamentalmente:

- Información personal del paciente: nombre y apellidos, datos de contacto, etc.

- Información sanitaria del paciente: tratamiento farmacológico relacionado con el riesgo vascular y problemas de salud relevantes con el control de la PA.
- Información sobre la medida y control de PA, junto con problemas detectados y medidas recomendadas si procede.

- Información sobre la derivación, si precisa, donde se indique el problema detectado y el servicio y al profesional al que se deriva.

\section{Calidad, certificación y mejora continua}

Con el fin de impulsar y garantizar la calidad de este servicio, SEFAC recomienda la inclusión de este servicio en un programa de aseguramiento de la calidad.

El requisito de la certificación tiene como objetivo atestiguar -a través de la emisión de un documento o certificado- que un servicio, persona o empresa se ajusta a unas normas técnicas de calidad determinadas.

Hay que distinguir entre tres tipos de certificaciones:

1. Certificación de profesionales: los farmacéuticos comunitarios que realicen el servicio de AMPA o MAPA deberán contar con la certificación de la capacitación de cada modalidad.

2. Certificación de establecimientos y equipos: las farmacias comunitarias que presten el servicio de AMPA o MAPA deberán disponer de la certificación de que se dispone de los recursos materiales necesarios para su prestación y en el caso de los equipos, que estén debidamente calibrados.

3. Certificación y validación de PNT: los PNT utilizados en la realización del servicio AMPA o MAPA deben haber sido validados para su utilización en la práctica clínica.

SEFAC ofrecerá además a los farmacéuticos interesados participar en un programa de supervisión externa de la calidad con el fin de poder compararse con otras farmacias y poder mejorar este servicio.

Independientemente de ello, se recomienda realizar periódicamente auditorías del servicio, así como encuestas de satisfacción a los pacientes $\mathrm{y}$ a los profesionales que lo prestan.

\section{Resultados obtenidos}

a) Las ventajas para el paciente de autoimplicarse en el control de su 


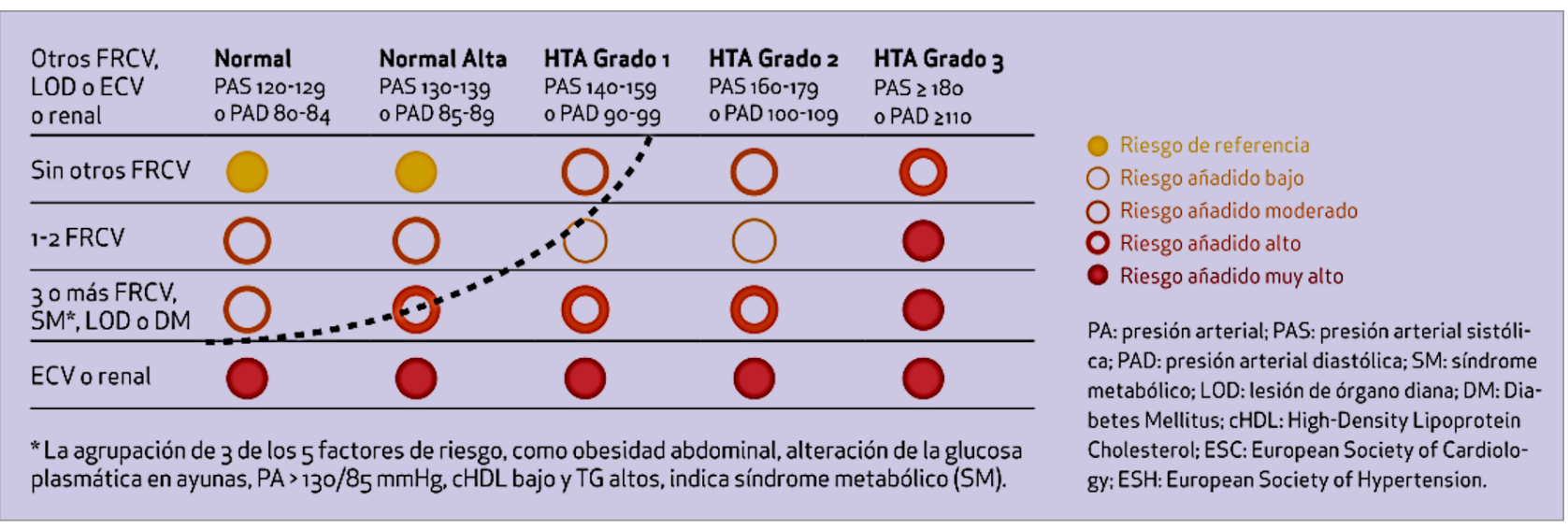

Figura 1 Estratificación del RV

HTA le suponen [6] mejorar el conocimiento sobre su PA [11] y concienciarse de la importancia de la toma de la medicación, lo cual puede llegar a mejorar la adherencia $[7,11,13]$ $\mathrm{y}$, en consecuencia, sus valores de presión arterial. La finalidad es situar al paciente dentro de los límites tensionales objetivos con el menor riesgo añadido posible para su situación clínica, tal como se muestra en la figura 1, todo ello derivado de la mejora de la interacción con su farmacéutico.

Diversos estudios en España demuestran que la implicación del farmacéutico en el control de la PA del paciente hipertenso mejora los valores hasta $6,8 \mathrm{~mm} \mathrm{Hg}$ en PAS y 2,1 mm Hg en PAD [14]. Otros estudios españoles [15] e internacionales [16] apuntan en la misma dirección.

Un servicio análogo de control de la PA en las farmacias comunitarias australianas muestra que el servicio continuado de control de la PA puede ayudar en seis meses a disminuir alrededor de $10 \mathrm{mmHg}$ la PAS de los pacientes receptores del servicio respecto de los que no lo reciben e incluso llega a $13 \mathrm{mmHg}$ la disminución en el subgrupo de pacientes atendidos que no mantenían una adherencia al tratamiento al principio de la intervención. Este resultado por sí solo justifica el servicio farmacéutico [17].

A su vez, estudios realizados en las farmacias comunitarias españolas confirman los resultados, resaltando la mejora de los valores tensionales de los pacientes atendidos por este servicio [18].

b) El farmacéutico incrementa su satisfacción profesional: el AMPA/MAPA le proporciona un mejor conocimiento de los valores tensionales del paciente, tanto del periodo de actividad como del periodo de descanso (en el caso de MAPA), lo cual permite conocer entre otras cosas la eficacia del tratamiento antihipertensivo, efecto bata blanca, necesidad del tratamiento (vital para el diagnóstico según las actuales guías clínicas) y facilita una mejor relación con otros profesionales sanitarios, tal como se describe en los estudios australianos ya mencionados.

c) Al médico le permite tener una visión más exacta de los valores de tensión arterial del paciente, lo cual le permite realizar un diagnóstico más exacto del paciente, una valoración de la efectividad de la medicación (mediante realización de controles posteriores).

d) $\mathrm{Al}$ sistema sanitario le aporta ahorro económico ya que permite conocer de forma exacta la necesidad de medicación, realización de diagnóstico de forma segura y eficiencia de la medicación. En el caso australiano [17], que es el caso que más datos aporta sobre la aplicación de este servicio en farmacia comunitaria se considera altamente coste-efectivo, ya que permite una ganancia en términos de años de vida ajustados por calidad (AVAC) de 6.322,58 AU\$, (4.489 €) superando ampliamente el límite de 700 AU\$ (495 €) fijado por el gobierno australiano para acciones costo-efectivas.

En tanto en cuanto sea técnicamente posible SEFAC permitirá que los interesados puedan participar en su programa de supervisión externa de la calidad con el fin de poder conocer los resultados obtenidos, compararse con otras farmacias y poder mejorar.

\section{Retribución y en su caso financiación}

\section{Retribución}

La forma de retribución recomendada para este servicio es aquella que cubra los costes del servicio, así como un honorario profesional libre establecido para su prestación en la farmacia comunitaria.

La realización de este servicio en la farmacia comunitaria conlleva una serie de costes que requieren ser cubiertos para que se pueda garantizar su viabilidad en el tiempo. Por tanto, la forma de retribución establecida debe al menos cubrir los costes que ocasiona su realización. Hay que tener en cuenta que su implementación puede suponer al farmacéutico comunitario una gran inversión económica, por diferentes motivos como la necesidad de un cambio en la estructura de la farmacia, con la creación de zonas de atención personalizada, la utilización de otros aparatos, un mayor número de nuevas herramientas informáticas, el entrenamiento del personal o incluso contratar más personal. Por otro lado supone un consumo adicional de tiempo (para la toma de datos con el paciente, estudio, toma de decisiones, diversos registros, etc.) con lo que esto supone de un mayor coste. En definitiva, para establecer la retribución de este servicio hay que determinar en primer lugar los costes y posteriormente el beneficio que se pretende obtener.

Los costes requeridos para su prestación incluyen:

1. Costes fijos (no varían con el número de pacientes atendidos): incluyen los costes derivados de la amortización del aparataje que se ha de adquirir para la realización del servicio: tensiómetros (con los tres diferentes manguitos disponibles, según 
perímetro del brazo del paciente) y en su respectiva proporción de: las inversiones realizadas para certificar este servicio (instalaciones, equipamiento y su mantenimiento), de los costes proporcionales de los gastos generales de la farmacia (luz, limpieza, acceso a internet, software, seguros, aplicación LOPD, etc.) y de la capacitación del personal. El coste de adquisición del aparataje (clínicamente validado) para AMPA y MAPA es:

- AMPA sobre $100 €$ en el año 2013, en función del modelo y marca seleccionado.

- MAPA sobre 1.000-2.000 $€$ en el año 2013, en función del modelo y marca seleccionado. En este sentido la MAPA tiene la desventaja de su elevado coste si bien es el método de valoración más exhaustivo puesto que describe la evolución de la PA durante 24 o 48 horas.

2. Costes variables o gastos de funcionamiento del servicio: incluyen los costes del material fungible (papel, tinta, etc.), cartelería, folletos, hojas para registros, baterías de los tensiómetros, archivo y la documentación necesaria, así como la calibración de los aparatos anualmente, los gastos de seguros de responsabilidad civil y de los aparatos.

3. Coste del profesional que el realiza el servicio, que depende del tiempo empleado. Este servicio siempre será prestado por un farmacéutico.

En función de la modalidad varían los tiempos:

MAFC: la medición entre 5-10 minutos en función del número de medidas efectuadas.

AMPA/MAPA: el tiempo medio de estos dos servicios suele estar en torno a los 45-60 minutos en la sesión inicial, siendo 20-30 minutos la media de entrevista con el paciente y el resto de estudio del caso y preparación del informe.

En el caso del AMPA/MAPA existen visitas sucesivas de seguimiento donde se estima un tiempo dedicado de 15-25 minutos, salvo que existan incidencias.

El coste económico relacionado con el tiempo empleado por el farmacéutico se ha calculado en referencia al coste laboral por hora de un farmacéutico, que puede variar según el convenio actual vigente al que esté sujeto la farmacia, entre 16,97 y $22,52 € /$ hora.
Como ejemplo, un servicio similar prestado en Australia por farmacéuticos comunitarios eleva el coste del servicio a unos 25 AU\$ $(17,70 €)$ por visita y paciente [20].

\section{Financiación}

El pago de este servicio, al igual que el resto de los SPF puede conjugar diferentes modalidades:

- pago íntegro por el paciente,

- cobertura total por sanidad pública/seguro privado,

- copago del paciente con cobertura parcial de la sanidad pública/seguro privado.

Dados los costes que supone el descontrol de la PA por su gran influencia en numerosas enfermedades y por los estudios realizados en otros países como es el caso australiano, este servicio es coste-efectivo [20,21] y estaría justificado que fuese financiado, total o parcialmente, de forma selectiva para determinados pacientes. Por los mismos motivos, este servicio puede reunir fácilmente las condiciones necesarias para que sea incluido en la cartera de servicios de las aseguradoras privadas.

\section{Referencias bibliográficas}

1. Baixauli Fernández VJ, Satué de Velasco E, Gil García MI, Roig Sena JC, Villasuso Cores B, Sáenz de Buruaga Pérez de Atxa S. Propuesta de la Sociedad Española de Farmacia Comunitaria (SEFAC) sobre servicios profesionales farmacéuticos en farmacia comunitaria. Farmacéuticos Comunitarios 2013; 5(3):119-26.

2. GIAF-UGR, SEFAC y SEH-LELHA. Guía de actuación para el farmacéutico comunitario en pacientes con HTA y riesgo cardiovascular. Documento de consenso. Farmacéuticos Comunitarios 2011; 3(2):69-83.

3. MacMahon S, Peto R, Cutler J, Collins R, Sorlie P, Neaton J, et al. Blood pressure, stroke, and coronary heart disease. Part 1. Prolonged differences in blood pressure: prospective observational studies corrected for the regression dilution bias. Lancet. 1990; 335:765-74. doi:10.1016/01406736(90)90878-9

4. Catalá López F, Sanfélix Gimeno G, García Torres C, Ridao M, Peiró S. Control of arterial hypertension in Spain: a systematic review and meta-analysis of 76 epidemiological studies on 341632 participants. Journal of Hypertension 2012; 30(1):168-76.
doi:10.1097/HJH.0b013e32834d30ec 5. National Institute for Health and Clinical Excellence (NICE) [Internet]. Hypertension: the clinical management of primary hypertension in adults. Clinical Guideline [CG127]. London: National Institute for Health and Clinical Excellence; 2011 [Acceso 20/08/2013]. Disponible en: www. nice.org.uk/guidance/CG127

6. Mancia G and Task Force Members. 2013 ESH/ESC Guidelines for the management of arterial hypertension. The Task Force for the management of arterial hypertension of the European Society of Hypertension (ESH) and of the European Society of Cardiology (ESC). Journal of Hypertension 2013; 31:1281-357. doi:10.1097/01. hjh.0000431740.32696.cc

7. Fikri Benbrahim N, Faus MJ, Martínez Martínez F, Sabater Hernández D. Impact of a community pharmacists' hypertension-care service on medication adherence. The AFenPA study. Res Social Adm Pharm. 2013 Feb 4. doi:10.1016/j.sapharm.2012.12.006. [Epub ahead of print] PubMed PMID: 23391845.

8. Banegas JR. Epidemiología de la hipertensión arterial en España. Situación actual y perspectivas. Hipertensión. 2005; 22:353-62. doi:10.1016/ S0212-8241(05)71587-5

9. Divison Garrote JA y grupo AMPA de SEH-LEHLA. Medidas domiciliarias de presión arterial. Documento de consenso. SEH-LELHA 2014. Hipertens Riesgo Vasc. 2015;32(1):27-39. doi:10.1016/j.hipert.2014.10.001

10. Iracheta M, Martínez S, Molinero A. ImpacHta: Formación SEFAC-SEH-LELHA en hipertensión y riesgo vascular. Madrid: SEFAC; 2014.

11. Quinto Grupo de Trabajo de la Sociedad Europea de Cardiología y otras Sociedades sobre la Prevención de la Enfermedad Cardiovascular en la Práctica Clínica. Guía europea sobre prevención de la enfermedad cardiovascular en la práctica clínica. Rev Esp Cardiol. 2012; 65(10):937.e1-e66.

12. Consejo General de Colegios Oficiales de Farmacéuticos [Internet]. BotPlus. Base de datos del conocimiento sanitario. [Acceso: 20/08/2013]. Disponible en http://www.portalfarma. com/inicio/botplus20/Paginas/BotPLUS-2-0.aspx

13. Lee JK, Grace KA, Taylor AJ. Effect of a pharmacy care program on medication adherence and persistence, blood pressure, and low-density lipoprotein cholesterol: a randomized controlled trial. JAMA. 2006 Dec 6; 296(21):2563-71. Epub 2006 Nov 13. PubMed PMID: 17101639. http:// dx.doi.org/10.1001/jama.296.21. joc60162 
14. Fikri Benbrahim N, Faus MJ, Martínez Martínez F, Alsina DG, Sabater Hernández D. Effect of a pharmacist intervention in Spanish community pharmacies on blood pressure control in hypertensive patients. Am J Health Syst Pharm. 2012 Aug 1; 69(15):131118. doi:10.2146/ajhp 110616

15. Torres A, Fité B, Gascón P, Barau M, Guayta Escolies R, Estrada Campmany $\mathrm{M}$ et al. Efectividad de un programa de atención farmacéutica en la mejora del control de la presión arterial en pacientes hipertensos mal controlados. Estudio PressFarm. Hipertens Riesgo Vasc. 2010; 27(1):1322. doi:10.1016/j.hipert.2009.05.007

16. Wentzlaff DM, Carter BL, Ardery G, Franciscus CL, Doucette WR, Chrischilles EA et al. Sustained blood pressure control following discontinuation of a pharmacist intervention. J Clin Hypertens (Greenwich). 2011 Jun; 13(6):431-7. doi:10.1111/ j.17517176.2011.00435.x. Epub 2011 Mar 18. PubMed PMID: 21649843.

17. Stewart K, George J, Jackson SL, Peterson GM, Hughes JM, Mc Namara KP, Bailey M, Hsueh A, McDowell J, Bereznicki L, Gee P. Increasing community pharmacy involvement in the prevention of cardiovascular disease. Canberra: Australian Government Department of Health and Ageing; 2010. 18. Penín Álvarez 0, Blanco González I, Villasuso Cores B. Resultados preliminares de una cohorte de hipertensos incluidos en un programa de MAPA en farmacia comunitaria en colaboración con el médico de AP. Congreso de la Sociedad Gallega de HTA. La Toja (Pontevedra) 2013.

19. Hughes JM, Keen N, Dillon M, Maricic T. Hypertension: Improving patient compliance and clinical outcomes through community pharmacist managed care. Canberra: Australian Government Department of Health and Ageing; 2005.

20. Mar J. Análisis de costes de la monitorización ambulatoria de la presión arterial en hipertensión leve. Gaceta Sanitaria 1999; 13(5):384-90. doi:10.1016/S0213-9111(99)71389-0

21. Llisterri JL. Aportación del proyecto CARDIORISC al manejo del paciente hipertenso de Atención Primaria en España. SEMERGEN-Medicina de familia. 2007; 33(9):495-501. doi:10.1016/ S1138-3593(07)73951-5 